\title{
Dimensiones de Resiliencia en los desplazamientos internos en la ciudad de Chilpancingo, Guerrero, México.
}

\author{
Dimensions of Resilience in internal displacement in the city of \\ Chilpancingo, Guerrero, Mexico.
}

\author{
Sotelo Leyva, José Francisco ${ }^{1}$ \\ ${ }^{1}$ Universidad Autónoma de Guerrero, Facultad de Arquitectura y Urbanismo, \\ Chilpancingo, Guerrero, México \\ 1jfsotelo@uagro.mx / https://orcid.org/0000-0002-4415-0268
}

Recibido el 15 de octubre de 2020, aprobado el 09 de diciembre de 2020

\begin{abstract}
RESUMEN | En las últimas décadas diversas ciudades de la República Mexicana han servido como receptoras de desplazamiento interno debido a contextos de violencia, conflicto y desastres de origen natural y humano, desgraciadamente la respuesta del estado ante el desplazamiento ha sido ineficaz y lento. Esta investigación surge de la necesidad de establecer estrategias y políticas a nivel local sobre la atención y recepción adecuada a poblaciones desplazadas por violencia o desastres, critica la manera en la cual se ha desarrollado el actuar del Estado Mexicano en la ciudad de Chilpancingo, Guerrero con respecto a la atención al desplazado en el momento de solicitar refugio, y finalmente propone cinco dimensiones sistémicas que servirán para aumentar por un lado la resiliencia de la ciudad como refugio y al desplazado como ser vulnerable.
\end{abstract}

PALABRAS CLAVE | Desplazamiento interno, Resiliencia, Atributos, Violencia y Desastre, Ciudad

\begin{abstract}
In recent decades, various cities in the Mexican Republic have served as recipients of internal displacement due to contexts of violence, conflict and disasters of natural and human origin, unfortunately the state's response to displacement has been ineffective and slow. This research arises from the need to establish strategies and policies at the local level on the adequate attention and reception of populations displaced by violence or disasters, it criticizes the way in which the actions of the Mexican State have developed in the city of Chilpancingo, Guerrero with regarding care for the displaced at the time of requesting refuge, and finally proposes five systemic dimensions that will serve to increase, on the one hand, the resilience of the city as a refuge and the displaced as a vulnerable being.
\end{abstract}

KEYWORD | Internal displacement, Resilience, Attributes, Violence and Disaster, City 


\section{Introducción}

En el 2019 ocurrieron 33.4 millones de nuevos desplazados por violencia o desastres en el mundo según el Reporte Global de Desplazamiento Interno elaborado por el IDMC (Centro de Monitoreo de Desplazamiento Interno). Este reporte enfatiza la creciente necesidad de que las ciudades se preparen y adopten esquemas de resiliencia más eficientes para acoger a poblaciones y comunidades desplazadas y brindarles un alto apoyo humanitario (The World Bank, 2017).

Sin embargo, muchas ciudades receptoras no han podido afrontar de manera adecuada los desplazamientos forzados y los resultados y estrategias han resultado en falacias y ocurrencias por la falta de preparación en su desarrollo en general. Esta investigación está dividida en dos partes la primera se enfoca principalmente a las intervenciones del Estado Mexicano como "ente" generador de resiliencia y de bienestar social en la ciudad de Chilpancingo, Guerrero, y la segunda; la propuesta principal de esta investigación que contempla una visión holística de los atributos y dimensiones de resiliencia que deben de adoptar las ciudades receptoras de desplazamiento con el fin de tener estrategias dignas y humanas para la población desplazada.

\section{Desarrollo.}

\section{EI desplazamiento interno; causas y las consecuencias.}

Los desplazamientos internos se definen como un proceso derivado de un grupo de personas altamente vulnerables obligadas a huir dentro de su propia frontera, $\mathrm{y}$ en la cual las causas pueden ser muy diversas, en el 2019 la cifra de desplazados internos en el mundo fue de 33.4 millones de nuevos casos (la cifra más alta después del 2012) de los cuales 8.5 millones fueron de conflicto y violencia y 24.9 millones por desastres (IDMC, 2019, pág. 4).

Las causas varían de país a país, pero la que más predomina son los conflictos armados la violencia comunitaria y los desastres relacionados con el clima (figura 1 y 2).

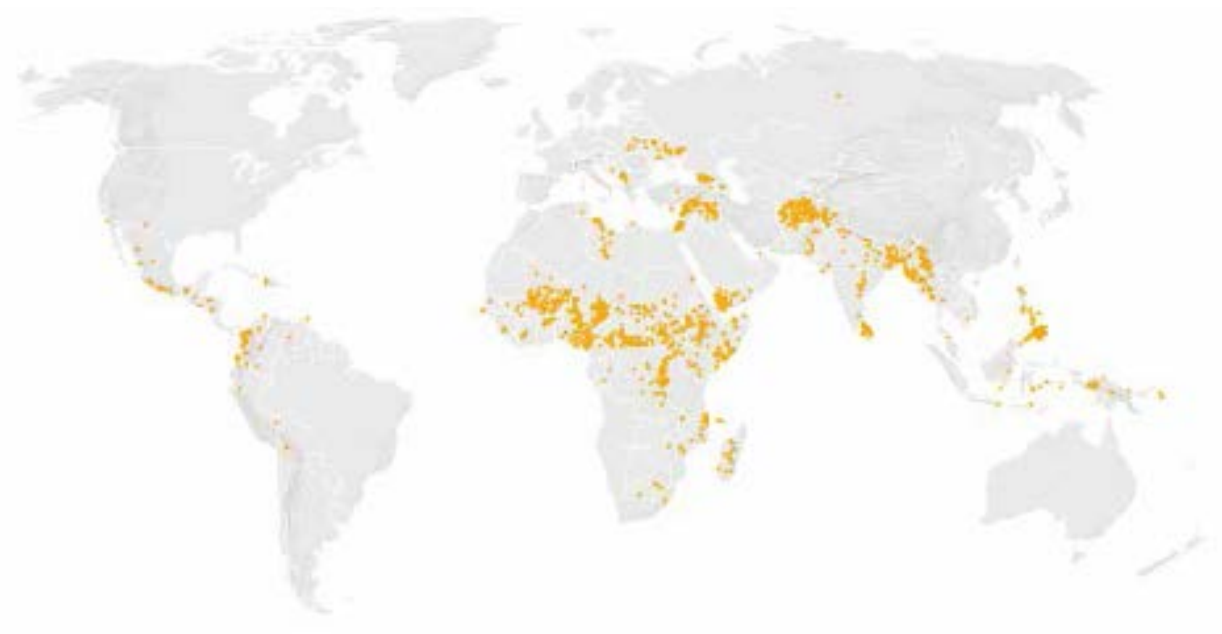

Figura 1: Ubicación de los desplazamientos por conflicto en el 2019. Fuente: IDMC, 2019. 


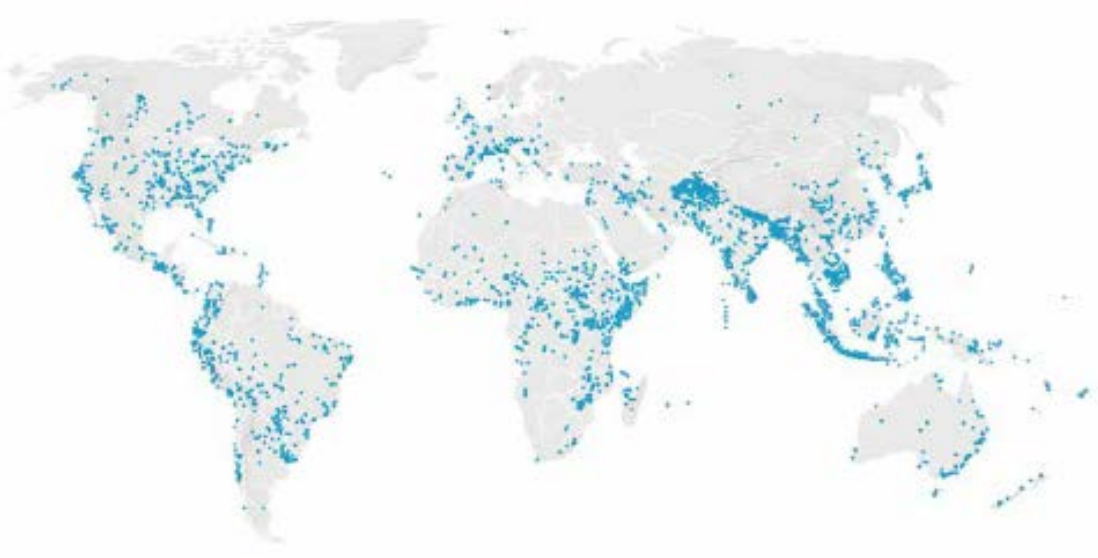

Figura 2: Ubicación de los desplazamientos por desastre en el 2019. Fuente: IDMC, 2019.

El concepto de desplazamiento interno ha sido comúnmente confundido con otros conceptos similares como son;

Migrantes: Son aquellos que deciden trasladarse de un lugar a otro no a una causa de amenaza directa de persecución o muerte sino para mejorar sus vidas al encontrar trabajo, por educación o reunión familiar entre otras causas (Edwards, 2020).

Refugiados: Son aquellos a quienes se les ha otorgado protección bajo la convención sobre el estatuto de los refugiados adoptada en Ginebra Suiza en 1951 u otras formas de protección complementaria o de manera temporal.

Solicitantes de Asilo: Se refiere a aquellos que han presentado solicitud de condición de refugiado en un país en particular y están en espera que el Estado decida sobre dicha solicitud (Sydnor, 2011).

Para el autor desplazamiento interno se define como un grupo de personas que puede ser integrado por familias o de forma individual obligadas a ser desplazadas en su propio territorio por un evento o fenómeno socio natural que deriva en un desastre, o bien por eventos de violencia diversa que los hace altamente vulnerable a perder la vida o bienes económicos.

El panorama para el futuro es poco halagador, ya que aún existen millones de personas que fueron desplazadas en años anteriores y que aún no han encontrado el soporte necesario para reconstruir su vida, llama la atención los 33,000 desplazados una década después del terremoto de Haití o los 1.2 millones de las inundaciones en Afganistán (IDMC, 2019). Las últimas estimaciones del IDMC países como Estados Unidos, El Salvador, Brasil y Bolivia fueron los países que más desplazamientos ocurrieron en el 2019 en las Américas debido a desastres por tormentas, incendios y violencia.

\section{Los desplazamientos en México; entendiendo el fenómeno.}

El fenómeno del desplazamiento en México no es nuevo, algunas investigaciones han demostrado que los conflictos y guerras en la cultura maya y mexica debido a construcciones de poder han movilizado a sociedades que históricamente fueron cautivas para rituales y sacrificios, sacralizando 
así la guerra como elemento jerárquico basado en la figura de un gobernante (Sánchez \& Risiglione, 2007). A su vez algunas culturas mayas dedicadas a la agricultura tuvieron que abandonar sus cetros en el valle y subir hacia las colinas, donde podía fortificarse de manera eficaz de los ataques de sus enemigos (Wolf, 2009).

Ya en la etapa contemporánea en la década de los setenta se inicia principalmente por problemas religiosos, problemas comunales o por disputas de tierras. En los noventa los desplazamientos fueron iniciados por problemas relacionados a conflictos armados como el de Chiapas y el conflicto Zapatista (CMDPDH, 2014, pág. 12). En la actualidad el fenómeno del desplazamiento se ha desarrollado por dos causas principales: el primero debido a desastres por fenómenos naturales y antrópicos y el segundo debido a desplazamientos por situación de violencia causada por los cárteles de narcotráfico principalmente en estados de Michoacán, Guerrero, Chiapas, Chihuahua, Tamaulipas y Sinaloa.

El IDMC precisa que en México en el año 2019 ocurrieron 7,100 desplazados por conflicto y violencia y 16,000 por desastres, (ver grafica 1). En primer lugar, se encuentra el estado de Guerrero con 5,056 personas desplazadas, y en segundo lugar Chiapas con 5035, seguido de Sinaloa, Oaxaca y Michoacán.

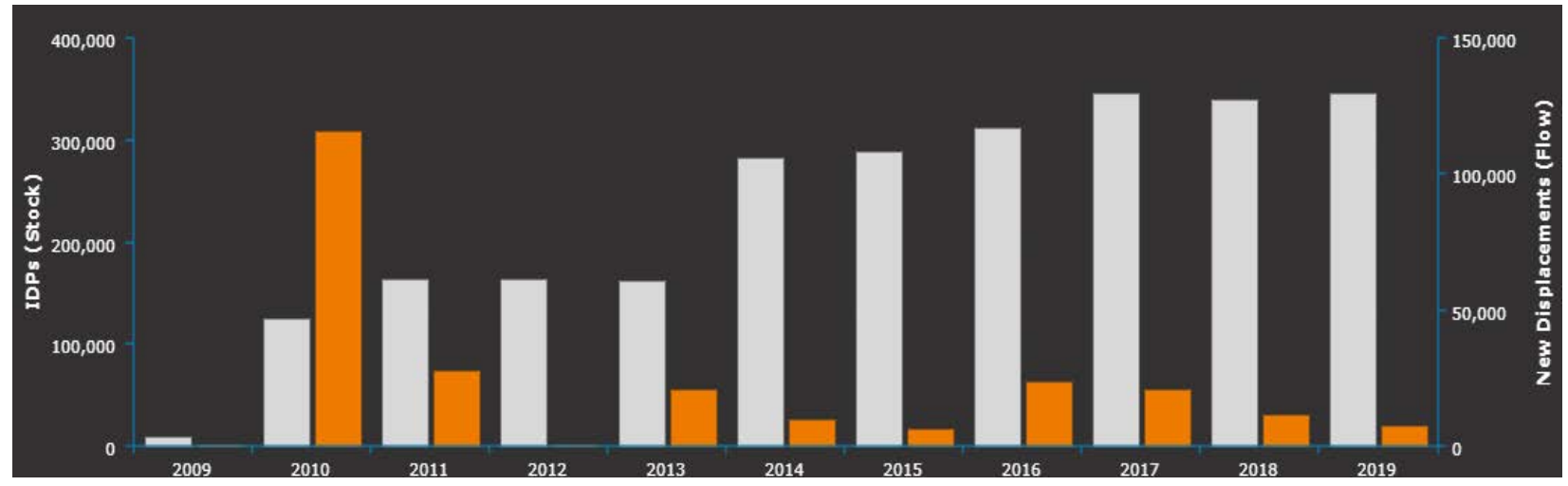

Gráfica 1: Muestra los acumulados de desplazamientos por año del 2009 al 2019, Fuente; http://internal-displacement. org/countries/mexico. Fecha de consulta; octubre 2020.

De los 28 episodios de desplazamientos en el 2019 en México, en 18 de ellos predominaron niños y niñas en un $64.29 \%$ mientras que en 12 episodios el $42.86 \%$ fueron mujeres, en 10 episodios el $35.71 \%$ fueron hombres y en 6 episodios $21.43 \%$ fueron adultos mayores (CMDPDH, 2020).

\section{El modelo de ciudad refugio en México.}

Una dificultad inicial sobre el concepto de ciudad refugio es que no existe una definición concreta en la literatura, gran parte de la información se encuentra dispersa en; a) en la población y las causas de su desplazamiento (IDMC, 2019), (CMDPDH, 2020) b) infraestructura urbana y vivienda (Araya, Faust, \& Kaminsky, 2020), (Kelman, Ashmore, Leon, \& D' urzu, 2014) y c) dimensiones sociales y culturales de los desplazados (Mercer, y otros, 2012), (Wang, Feng, Han, Zuo, \& Rameezdeen, 2020).

En México la experiencia de generar resultados positivos con respecto a los desplazamientos internos ha tenido en su mayoría resultados negativos, como bien lo precisa el Centro de Monitoreo de Desplazamiento Interno (IDMC por sus siglas en inglés) 16,000 personas han sido desplazadas de su lugar de origen por desastres de origen socio natural y 7,100 por conflicto y violencia en 
México, es así que surge la necesidad de establecer políticas de resiliencia para personas que en su mayoría han perdido bienes económicos, familia y vivienda. Aunado a esto la necesidad de salir a buscar refugio porque al perder todo buscan la protección y el abrigo que el Estado les puede otorgar.

Pero el problema reside cuando el Estado no está preparado para este tipo de dificultades, queda aletargado y sumido en una total incertidumbre con respecto al shock que pueda ocasionar la llegada de población en busca de ayuda y refugio, la gráfica 2 denominada "Gráfica de resiliencia" nos muestra que a través del tiempo las ciudades pueden sufrir un shock (desastres, guerra, violencia, desplazamiento etc.) la ciudad entra en una etapa de inactividad (incertidumbre) y depende de que tan fortalecida se encuentre (resiliencia) podrá salir y recuperarse adecuadamente.

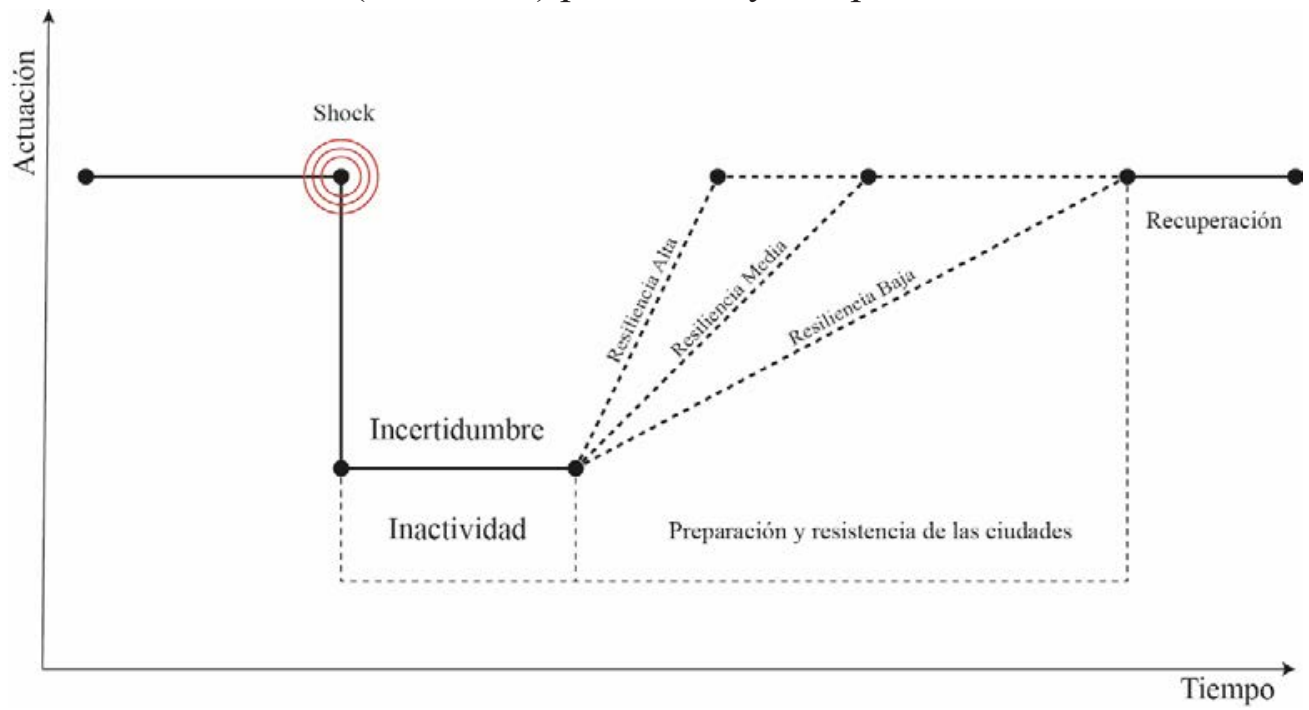

Gráfica 2: Gráfica de resiliencia, Fuente; Modificado de (Tierney, 2002), (2020).

\section{La intervención del Estado en la ciudad de Chilpancingo, Guerrero.}

En la ciudad de Chilpancingo, Guerrero la actuación ante este tipo de eventos se ha realizado de forma muy errada en su gran mayoría, principalmente por la falta de preparación en la actuación de un evento perturbador (violencia o desastre) (figura 3).

Cabe mencionar casos como el del Nuevo Mirador en la ciudad de Chilpancingo, Guerrero, México (desplazamiento en la misma ciudad por desastre de origen socio natural) (figura 4 y 5) y el de familias de comunidades del municipio de Leonardo Bravo en el estado de Guerrero, México por violencia y conflicto (figura 6).

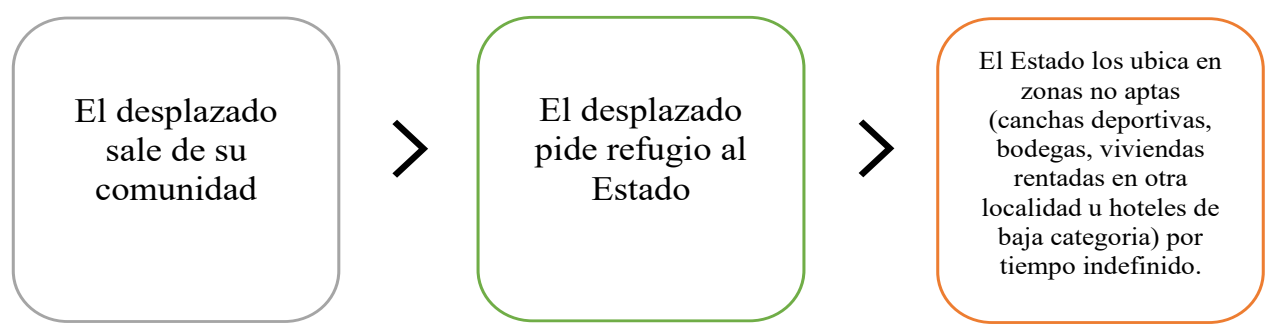

Figura 3: Ciclo de atención del Estado a los desplazados en la ciudad de Chilpancingo, Guerrero, México, Fuente: Elaboración del autor, 2020. 


\section{Proyecto Nuevo Mirador.}

Proyecto habitacional elaborado a partir del desastre de la tormenta tropical Ingrid y el Huracán Manuel el día 15 de septiembre del 2013, el proyecto se caracteriza por fallas en la forma de abordar el tema cultural de la vivienda, fallas en la elección del sitio de reubicación y alta corrupción en su ejecución.
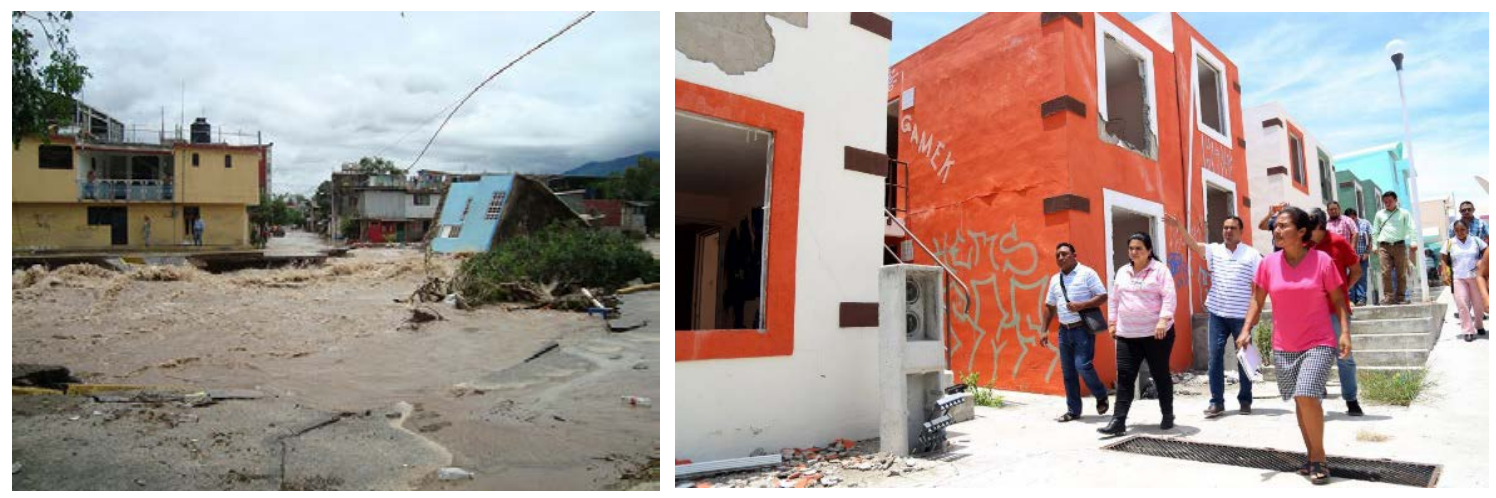

Figura 4 y 5: viviendas construidas para reubicación permanente en el Nuevo Mirador en la Ciudad de Chilpancingo, Guerrero, México, Fuente; https://suracapulco.mx/demoleran-el-viernes-edificios-para-damnificados-en-el-miradoren-chilpancingo/

\section{Desplazamiento por violencia en comunidades del estado de Guerrero.}

56 familias desplazadas de sus comunidades en el municipio de Leonardo Bravo en el estado de Guerrero reubicadas en un poblado cercano a la ciudad capital del estado de Guerrero, el manejo de este desplazamiento no ha sido optimo, las personas dependen de lo que el estado les pueda garantizar (sustento económico principalmente) esto ha generado estrés emocional por los procesos de desterritorialización.

En ambos casos predominó la falta de preparación, la alta incertidumbre y la corrupción para no generar estrategias de resiliencia tanto para las ciudades como para la población vulnerable.

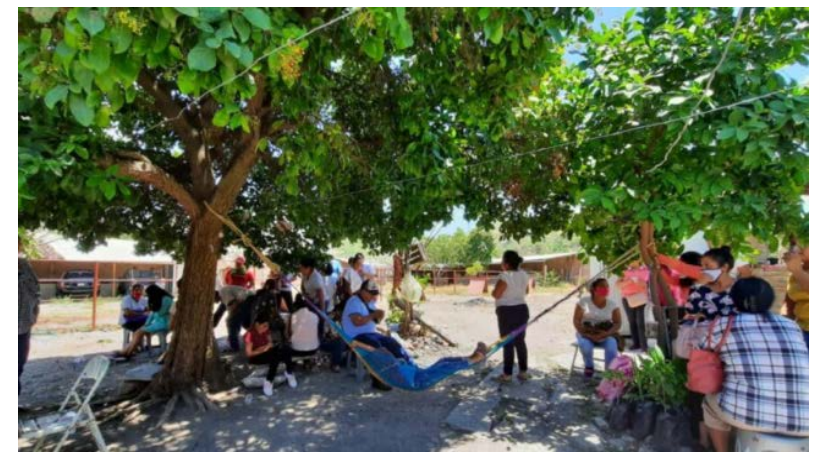

Figura 6: familias desplazadas de diferentes comunidades del municipio de leonardo Bravo en el estado de Guerrero, México, Fuente: https://www.elsoldechilpancingo.mx/2020/06/05/56-familias-desplazadas-dejaron-de-recibir-apoyodel-gobierno-federal-para-pagar-las-rentas-de-casas-en-chichihualco/ 


\section{Método}

\section{Atributos y dimensiones de la resiliencia en la creación de las ciudades refugio.}

Si bien el tema es complejo y difícil de abordar esta sección tratara de dar un acercamiento a que dimensiones se deben de abordar al momento de recibir a un desplazamiento en alguna ciudad receptora. Es aquí donde se tratará de adaptar algunos atributos de resiliencia para gestionar las ciudades y hacerlas más resistentes ante una perturbación o shock. Las investigaciones en este tema se basan es estudios de (Cutter, y otros, 2008), de (Burton, 2012), y de (Orencio \& Fujii, 2013).

Los estudios de Cutter y Burton mencionan la importancia de incorporar los atributos sociales, organizacionales, económicos y de infraestructura, Orencio y Fujii por su lado incorporan atributos de medio ambiente, protección social y criterios de planificación.

\section{Resultados}

Para efectos de esta investigación y como propuesta principal se han agregado algunos atributos o dimensiones que permitirán complementar los estudios de Cutter, Burton, Orencio y Fujii y que se puedan construir estrategias más cercanas a la población desplazada (figura 7).

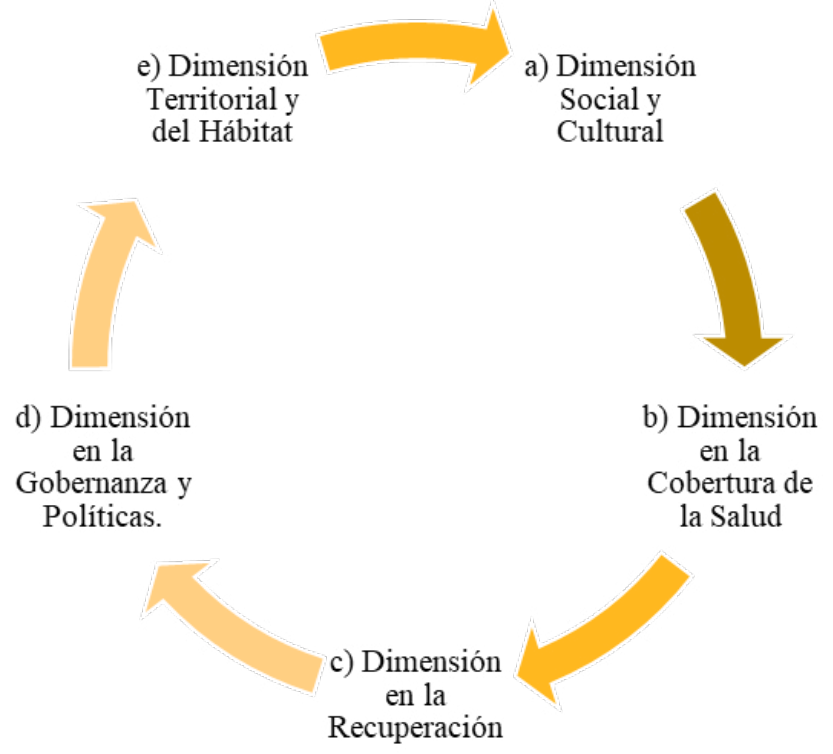

Figura 7: Dimensiones de las ciudades receptoras de desplazamiento Interno, Fuente; Elaboración del autor, (2020).

\section{Análisis de las dimensiones}

- Social y cultural.

Permite entender cómo se encuentra el desplazado con respecto a lo que le sucedió, o porque le sucedió, a su vez buscar fortalezas y soporte en su sistema de creencias que ofrezcan resiliencia mental y soporte ante las perspectivas de futuro, por ultimo el conocer sus grados de estudio permitirá dar información variada y personalizada a cada uno de ellos con respecto a los pasos siguientes, se recomienda que estos estudios lo realicen personal afín al campo de la sociología. 
- Cobertura en la salud.

Atención de primer contacto para saber que atención en la salud se les proporcionará, además los servicios de salud que asistan podrán tener estadísticas sobre que medicamentos utilizan, si tienen seguros particulares o laborales, así como un control sobre epidemias, vacunas y recomendaciones generales.

- Recuperación.

En esta dimensión es importante identificar las cualidades y fortalezas que tengan las personas desplazadas, buscando lideres en ellos para organizar por su cercanía a los grupos afectados, también se debe de analizar cual era su situación laboral en su comunidad o lugar de donde fue desplazado para poder reintegrarlo a empleos temporales o talleres en caso de tener conocimientos de algún oficio (carpintería, herrería etc.) por último analizar que tan factible es que regresen a su comunidad con ciertas reservas por ejemplo en caso de un desplazamiento por delincuencia organizada ver si el Estado puede darles acompañamiento y soporte en seguridad por tiempo ilimitado, o bien en caso de desastre puedan reubicarse de manera temporal cerca de su comunidad con facilidades expuestas en el punto de territorio y hábitat. Para el estudio de esta dimensión es necesario formar equipos multidisciplinarios como sociólogos, economistas y personal de gobierno entre otros.

- Gobernanza y Políticas.

Se considera que esta dimensión es la parte clave que promueve que las demás funcionen adecuadamente, es muy importante fomentar que en las partidas económicas destinar recursos y subsidios para atender el fenómeno del desplazamiento en las ciudades, por otro lado la interacción entre la seguridad y resguardo de las viviendas en caso de desastre (se entiende que puede existir robo de pertenencias) y el propio resguardo de las personas en el sitio temporal es de vital importancia para la tranquilidad de la población vulnerable. Es necesario que diversos actores tomen acciones para poder atender esta dimensión, por un lado, las políticas y cambios en la legislación promovidos por legisladores hasta la aplicación de programas con seguimiento de diversas ONG's.

- Territorio y Hábitat.

Es importante incluir en esta dimensión a la academia, a laboratorios y a personal con preparación especializada en estudios del territorio con características de hábitat y de diseño tecnológico en elaboración de viviendas. El diseño y estudio de una vivienda emergente permitirá tener un carácter mas humano con la población que generalmente es reubicada en una cancha deportiva, alguna bodega o en sitios no aptos para permanecer un tiempo determinado, permitirá tener un espacio con privacidad por el tiempo que sea necesario, además la vivienda con esta característica se podrá desmontar y llevar a otros lugares que se necesiten con la misma o similar emergencia, por ultimo es necesario analizar los sitios que serán para reubicaciones permanentes (en el caso de que el desplazamiento sea de la misma ciudad y que sea necesaria su reubicación por ejemplo destrucción de sus vivienda por un sismo). El estudio profesional urbanístico permitirá reubicar en zonas aptas tanto en las características urbanas de la propia ciudad y libres de riesgos según los últimos marcos internacionales en el tema (Metze) (Metzger \& Robert, 2013). 
Tabla 1: Dimensiones para formar resiliencia en la ciudad ante el desplazamiento, Fuente; Elaboración del autor (2020).

\begin{tabular}{|c|c|c|c|}
\hline Dimensión & Ejes Rectores & Descripción & Acciones \\
\hline \multirow{3}{*}{ Social y Cultural } & $\begin{array}{l}\text { Percepción y actitud hacia el } \\
\text { riesgo }\end{array}$ & $\begin{array}{l}\text { Es importante que el } \\
\text { desplazado entienda } \\
\text { el carácter social que } \\
\text { sucedió en su comunidad, } \\
\text { por ejemplo, si fue un } \\
\text { fenómeno natural sucedió } \\
\text { por ¿estar cerca de un } \\
\text { barranco? O bien si } \\
\text { fue un desplazamiento } \\
\text { por violencia preguntar } \\
\text { ¿cómo ellos entienden lo } \\
\text { que sucedió? }\end{array}$ & $\begin{array}{l}\text { A través de platicas, } \\
\text { reuniones y talleres con } \\
\text { personal especializado, } \\
\text { obtener respuestas de } \\
\text { preguntas tales como ¿Qué } \\
\text { piensan de lo que paso? } \\
\text { ¿Por qué sucedió? Etc. }\end{array}$ \\
\hline & Sistemas de creencias & $\begin{array}{l}\text { Dar soporte al desplazado } \\
\text { por medio de sus creencias } \\
\text { religiosas, filosóficas } \\
\text { o científicas (según el } \\
\text { caso) esto puede reforzar } \\
\text { su estado mental con } \\
\text { respecto a su sistema de } \\
\text { creencias. }\end{array}$ & $\begin{array}{l}\text { Ubicar de acuerdo con la } \\
\text { zona donde se encuentre } \\
\text { el desplazado a iglesias, } \\
\text { templos, institutos etc., } \\
\text { donde se le puede dar el } \\
\text { soporte analizado. } \\
\text { Tener estrategias con } \\
\text { ministros, curas, pastores, } \\
\text { o toda aquella persona } \\
\text { que puede dar apoyo a su } \\
\text { sistema de creencias donde } \\
\text { se encuentre el grupo de } \\
\text { desplazados. }\end{array}$ \\
\hline & Nivel de educación. & $\begin{array}{l}\text { Se refiere a entender que } \\
\text { tipo de educación ha } \\
\text { recibido el desplazado. }\end{array}$ & $\begin{array}{l}\text { Conocer si sabe leer y/o } \\
\text { escribir, esto es para } \\
\text { desarrollar estrategias } \\
\text { de cómo se les puede } \\
\text { dar información más } \\
\text { personalizada. } \\
\text { Si la persona presenta alto } \\
\text { soporte académico, pueda } \\
\text { servir como facilitador en } \\
\text { la información que se les } \\
\text { ofrece al grupo desplazado. }\end{array}$ \\
\hline $\begin{array}{l}\text { Cobertura en la } \\
\text { Salud }\end{array}$ & $\begin{array}{l}\text { Atención de primer contacto } \\
\text { con la población desplazada. }\end{array}$ & $\begin{array}{l}\text { Asistencia médica de } \\
\text { primer contacto para } \\
\text { personas vulnerables o de } \\
\text { enfermedades crónicas, } \\
\text { conocer su estado físico y } \\
\text { mental y llevar un registro } \\
\text { para futuros seguimientos. }\end{array}$ & $\begin{array}{l}\text { Brigadas de auxilio } \\
\text { mediante } \\
\text { programadas. } \\
\text { Canalización de personas } \\
\text { y orientación en caso de } \\
\text { epidemias o contagios. } \\
\text { Terapias alternas para } \\
\text { casos de depresión y } \\
\text { trastornos por causa del } \\
\text { desplazamiento. }\end{array}$ \\
\hline
\end{tabular}




\begin{tabular}{|c|c|c|c|}
\hline Dimensión & Ejes Rectores & Descripción & Acciones \\
\hline \multirow[t]{2}{*}{$\begin{array}{l}\text { Cobertura en la } \\
\text { Salud }\end{array}$} & Control de epidemias & $\begin{array}{l}\text { Poner en práctica las } \\
\text { medidas de salud de } \\
\text { las autoridades ante las } \\
\text { epidemias. }\end{array}$ & $\begin{array}{l}\text { A través de un control } \\
\text { sanitario con apoyo de } \\
\text { especialistas en la salud, ver } \\
\text { la forma en que habitaran } \\
\text { de manera temporal con } \\
\text { estrategias de sana distancia } \\
\text { y con estricto apego a los } \\
\text { protocolos sanitarios. } \\
\text { Buscar que se dé prioridad } \\
\text { a el uso de vivienda } \\
\text { emergente para aislamiento } \\
\text { en caso de contagio. }\end{array}$ \\
\hline & Acceso a asistencia médica & $\begin{array}{l}\text { Conocer de manera rápida } \\
\text { la condición médica de los } \\
\text { desplazados. }\end{array}$ & $\begin{array}{l}\text { canalizar o llevar un } \\
\text { seguimiento muy cercano } \\
\text { con aquellas personas } \\
\text { desplazadas que presenten } \\
\text { problemas mayores de } \\
\text { salud. }\end{array}$ \\
\hline \multirow{4}{*}{ Recuperación } & Nivel de resiliencia & \begin{tabular}{lr|}
\multicolumn{2}{l}{ Se refiere a las fortalezas } \\
personales & que el \\
individuo & desplazado \\
pueda tener. &
\end{tabular} & $\begin{array}{lr}\begin{array}{l}\text { Conocer las } \\
\text { personales, }\end{array} & \text { físicas, } \\
\text { mentales y } & \text { emocionales } \\
\text { de los desplazados } & \text { elas } \\
\text { entre ellas se puede } \\
\text { considerar su sistema de } \\
\text { creencias, pensamientos y } \\
\text { perspectivas de futuro. }\end{array}$ \\
\hline & Situación laboral & $\begin{array}{l}\text { Conocer a que se dedicaba } \\
\text { en su comunidad o } \\
\text { localidad del cual fue } \\
\text { desplazado. }\end{array}$ & $\begin{array}{l}\text { Esto servirá } \\
\text { incorporarlo a para } \\
\text { trabajo temporal y tener } \\
\text { la oportunidad de ser } \\
\text { productivo. }\end{array}$ \\
\hline & Acceso a seguros financieros & $\begin{array}{l}\text { Se refiere a que servicios } \\
\text { financieros } \\
\text { oportunidad de acceder. }\end{array}$ & $\begin{array}{l}\text { Por ejemplo, facilidades a } \\
\text { seguros de vida, educativos } \\
\text { o incluso de manutención. }\end{array}$ \\
\hline & $\begin{array}{l}\text { Factibilidad de regreso a su } \\
\text { comunidad }\end{array}$ & $\begin{array}{l}\text { Analizar si es factible el } \\
\text { regreso a su comunidad } \\
\text { por medio de acciones } \\
\text { correctivas. }\end{array}$ & $\begin{array}{l}\text { Es necesario conocer } \\
\text { de manera puntual que } \\
\text { sucedió en la comunidad } \\
\text { en ocasiones algunos } \\
\text { d e s p l a z a m i e n t o s } \\
\text { se resuelven r con } \\
\text { acompañamiento r y } \\
\text { asignación de seguridad en } \\
\text { sus comunidades. }\end{array}$ \\
\hline
\end{tabular}




\begin{tabular}{|c|c|c|c|}
\hline Dimensión & Ejes Rectores & Descripción & Acciones \\
\hline \multirow{3}{*}{$\begin{array}{l}\text { Gobernanza y } \\
\text { políticas }\end{array}$} & Vigilancia y resguardo & $\begin{array}{l}\text { Resguardo y vigilancia } \\
\text { de los bienes materiales } \\
\text { y económicos en sus } \\
\text { viviendas (en casos en los } \\
\text { cuales sea factible, por } \\
\text { ejemplo, una inundación } \\
\text { leve) } \\
\text { Resguardo de la integridad } \\
\text { física de los desplazados } \\
\text { (vigilancia y resguardo en } \\
\text { la zona donde encuentren } \\
\text { refugio). }\end{array}$ & $\begin{array}{l}\text { Recorridos y guardias en } \\
\text { la zona por parte de las } \\
\text { autoridades. Vigilancia en } \\
\text { el sitio de reunión. } \\
\end{array}$ \\
\hline & $\begin{array}{l}\text { Transparencia de la } \\
\text { información }\end{array}$ & $\begin{array}{l}\text { Generar mecanismos en } \\
\text { los cuales se les puede } \\
\text { informar el avance } \\
\text { y las soluciones a su } \\
\text { desplazamiento. }\end{array}$ & $\begin{array}{l}\text { Puede ser por medio de la } \\
\text { prensa o bien de alguna } \\
\text { ONG'S con carácter legal. }\end{array}$ \\
\hline & Fondos para apoyo financiero & \begin{tabular}{|ll} 
Partidas especiales \\
económicas para el \\
aumento de la resiliencia \\
ante los desplazamientos.
\end{tabular} & \begin{tabular}{|lll} 
Integrar una partida \\
financiera destinada al \\
rubro del desplazamiento \\
interno dentro del \\
presupuesto anual de la \\
ciudad receptora.
\end{tabular} \\
\hline \multirow{2}{*}{$\begin{array}{l}\text { Territorio y } \\
\text { Hábitat }\end{array}$} & Vivienda emergente & \begin{tabular}{l}
\multicolumn{2}{l}{ Vivienda emergente de } \\
característica temporal \\
para ubicar a los \\
desplazados.
\end{tabular} & $\begin{array}{l}\text { Programas de proyecto } \\
\text { y diseño de vivienda } \\
\text { emergente que se pueda } \\
\text { mover, transportar y } \\
\text { construir de manera rápida } \\
\text { que preserve la intimidad } \\
\text { y la dignidad de los } \\
\text { desplazados. } \\
\text { Esta estrategia puede ser } \\
\text { desarrollada con la ayuda } \\
\text { de universidades, ONG'S y } \\
\text { gobierno local. }\end{array}$ \\
\hline & $\begin{array}{l}\text { Análisis de las zonas de } \\
\text { reubicación }\end{array}$ & $\begin{array}{l}\text { Estudios urbanos donde se } \\
\text { analice el emplazamiento } \\
\text { temporal o permanente } \\
\text { (en caso de que sea el } \\
\text { desplazamiento en la } \\
\text { misma ciudad por un } \\
\text { desastre) }\end{array}$ & $\begin{array}{l}\text { Estudiar desde un ámbito } \\
\text { territorial o urbano las } \\
\text { zonas donde se reubicarán } \\
\text { de forma permanente el } \\
\text { análisis debe de contener: } \\
\text { Análisis de riesgos. } \\
\text { Movilidad con la ciudad. } \\
\text { Nivel de infraestructura y } \\
\text { equipamiento. } \\
\text { Pertinencia con las } \\
\text { estrategias internacionales } \\
\text { (UN- Hábitat, UNDRR } \\
\text { etc). }\end{array}$ \\
\hline
\end{tabular}




\section{Conclusiones.}

Sin duda el tema del aumento en el fortalecimiento y la resiliencia de las ciudades refugio o receptoras con respecto a los desplazamientos internos es muy complejo. Algunas experiencias han demostrado que es un proceso lento y que deben englobar enfoques holísticos y sistémicos en su implementación. Esta investigación propone precisamente el inicio y la construcción de las bases en la cual se puede fortalecer la ciudad receptora dando un trato humanitario a toda persona que pide refugio ya sea temporal o permanente. La creciente necesidad de encontrar soluciones a corto plazo es muy importante ya que las perspectivas en el futuro de los problemas de ocurrencias de desplazamiento van en aumento; cambio climático, clima cambiante y aumento en la temperatura de la tierra ocasionan ciclones y tormentas que provocan inundaciones a comunidades y a ciudades afectando el modo de vida de muchas personas, así como también los problemas de delincuencia organizada, narcotráfico y violencia en general hacen insostenible habitar zonas con alto peligro social.

La comprensión de estas dimensiones y la puesta en práctica de ellas en las ciudades receptoras dará la pauta para establecer y comprender las repercusiones directamente en la población desplazada puesto que será a través de indicadores de bienestar si el impacto es sobresaliente o bien se requiere mejorar, ampliar o construir otros atributos que refuercen esta primera etapa.

\section{Bibliografía}

CMDPDH. (2014). Desplazamiento Interno Forzado en México. DF, México: Comisión Mexicana de Defensa y Protección de los Derechos Humanos.

CMDPDH. (2020). Episodios de desplazamiento interno forzado masivo en México. CDMX: Taller de sueños.

Cutter, S., Barnes, L., Burton, C., Evans, E., Tate , E., \& Webb, J. (2008). A place-based model for understanding community resilience to natural disasters. Global Environmental Change, 598-606.

Araya, F., Faust, K., \& Kaminsky, J. (2020). Understanding hosting communities as a stakeholder in the provision of. Sustainable Cities and Society, 1-11.

Burton, C. (2012). The development of metrics for community resilience to natural disasters. California: University of South Carolina.

Edwards, A. (11 de 10 de 2020). www.acnur.org. Obtenido de https://www.acnur.org/noticias/ noticia/2016/7/5b9008e74/refugiado-o-migrante-cual-es-el-termino-correcto.html

IDMC. (2019). Global Report On Internal Displacement 2019. NRC.

Kelman, I., Ashmore, J., Leon, E., \& D' urzu, S. (2014). From research to practice (and vice versa) for post-disaster. Environmental Hazards, 262-278.

Mercer, J., Gaillard, J., Crowley, K., Shannon, R., Alexander, B., Day, S., \& Becker, J. (2012). Culture and disaster risk reduction: Lessons. Environmental Hazard, 74-95. 
Metzger, P., \& Robert, J. (2013). Elementos de reflexión sobre la resiliencia urbana: Usos criticables y aportes potenciales. Territorios, 21-40.

Orencio, P., \& Fujii, M. (2013). A spatiotemporal approach for determining disaster-risk potential based on damage consequences of multiple hazard events. Jornal of Risk Research, 815-836.

Sánchez, L., \& Risiglione, C. (2007). Conflicto y guerra en los estados mayas y mexica. Preguntas en torno a la construcción del poder. XI Jornadas Interescuelas Departamentos de Historia.

Sydnor, P. (2011). Understanding the Forced Displacement of Refugees in Terms of the Person. Transformation SAGE, 51-61.

The World Bank. (2017). Cities of refuge in the Middle East. IBRD-IDA.

Tierney, K. (2002). Conceptualizing and measuring organizational and community resilience: Lessons from the emergency response following the September 11, 2001 attack on the World Trade Center. University of Delaware.

Wang, Y., Feng, Y., Han, Q., Zuo, J., \& Rameezdeen, R. (2020). Perceived discrimination of displaced people in development-induced. Cities, 1-11.

Wolf, E. (2009). Pueblos y Culturas de Mesoamérica. México, DF: Biblioteca Era, Ensayo. 